\title{
Introducing Relativity in Global Navigation Satellite Systems
}

\author{
J.-F. Pascual-Sánchez \\ Dept. Matemática Aplicada, Facultad de Ciencias, \\ Universidad de Valladolid, Valladolid, 47005, Spain, EU.*
}

\begin{abstract}
Today, the Global Navigation Satellite Systems, used as global positioning systems, are the GPS and the GLONASS. They are based on a Newtonian model and hence they are only operative when several relativistic effects are taken into account. The most important relativistic effects (to order $1 / c^{2}$ ) are: the Einstein gravitational blue shift effect of the satellite clock frequency (Equivalence Principle of General Relativity) and the Doppler red shift of second order, due to the motion of the satellite (Special Relativity). On the other hand, in a few years the Galileo system will be built, copying the GPS system unless an alternative project is designed. In this work, it will be also shown that the SYPOR project, using fully relativistic concepts, is an alternative to a mere copy of the GPS system. According to this project, the Galileo system would be exact and there would be no need for relativistic corrections.
\end{abstract}

PACS numbers: 03.30.+p, 04.25.Nx, 04.80.Cc, 95.30.Sf 


\section{INTRODUCTION}

The general purpose of this work is to give a very simple, yet rigorous, mathematical derivation of the relativistic effects on Global Navigation Satellite Systems (hereafter GNSS), suitable as an introduction to the subject. Also, I will focus on the essential differences between a Newtonian plus relativistic corrections framework, as that of the current operating systems, and a fully relativistic framework which would be desirable to implement in the future Galileo system due to its theoretical and practical advantages.

At present, all the GNSS, functioning only as global positioning systems, are the GPS (Navstar) (USA) $\frac{1}{2}$ and the GLONASS (Russia) $)^{2}$. Both started as military oriented networks. Nowadays, there are many important civilian applications of the GNSS such as: positioning and timing; comparison of time standards around the world; movements of buildings and bridges that can be monitored in real time; measurements of relative movements of the parts of the Earth's crust to forecast earthquakes; location of missing persons; tests of Special and General Relativity, etc. As one can see, the civilian applications of the GNSS have overtaken military applications.

The main characteristics of the present GNSS will be described in Sec. II. They are based on a Newtonian model which is corrected numerically by numerous and important "relativistic effects". This Newtonian model uses a non-inertial spatial reference frame for navigation that co-rotates with the Earth and is geocentric: the ECEF (Earth Centered Earth Fixed system). In the GPS, the ECEF is the military reference system World Geodetic System1984, WGS-84. Moreover, this Newtonian model uses as time reference, the coordinate time of a local inertial "star-fixed" reference frame with origin in the Earth's center of mass and freely falling with it, the ECI (Earth Centered Inertial system). An introduction to the navigation equations will be given in Sec. III.

In general, the satellites of the GNSS are affected by Relativity in three different ways: in the equations of motion, in the signal propagation and in the beat rate of the satellite clocks. In this work I will focus on the clock effects because they are the only measurable ones in the present GNSS. Among the relativistic effects on the rate of clocks, the most important ones (to order $1 / c^{2}$ ) are: the Einstein effect or gravitational frequency shift of the satellite atomic clocks (Equivalence Principle of General Relativity) and the Doppler shift of second order, due to the motion of the satellites (Special Relativity). If they were not corrected, 
the system would not be operative after a few minutes. In a day of operation, they would produce an error of more than $11 \mathrm{~km}$ in the horizontal positioning of the receiver. In a week, the error accumulated in the vertical positioning would be of $5 \mathrm{~km}$ approximately.

I will describe these effects in Sec. IV by means of the first post-galilean approximation to Special Relativity and the first post-Newtonian approximation to General Relativity using the static spherically symmetric Schwarzschild's metric (or even using the spacetime metric induced by the Equivalence Principle in the particular case of circular orbits) for the Earth.

Nevertheless, a constellation of satellites equipped with clocks broadcasting their proper times by means of electromagnetic signals, is indeed a proper relativistic or Einstein system. According to the SYPOR (a French acronym for SYstème de POsitionnement Relativiste) project for the Galileo (EU) by Coll $3,4,5$, which is a fully relativistic way to understand a GNSS system, the Control Segment should be in the constellation of satellites and therefore coincide with the Space Segment and not in the terrestrial geoid as in the current systems. Thus, the usual reading would be inverted and the function of the Control Segment would not be to determine the position of the satellites or space vehicles (hereafter SVs) with regard to certain terrestrial coordinates, but to define these with respect to the constellation of satellites.

According to the SYPOR project the Galileo system would be exact and there would be no need for "relativistic" corrections. The main ideas behind this project will be discussed in the final Sec. V.

Globally, the current situation in the GNSS is almost analogous to the following one: imagine that a century after Kepler, the astronomers were still using Kepler's laws as algorithms to correct the Ptolemaic epicycles by means of "Keplerian effects". Similarly, a century after Einstein, one still uses the Newtonian theory and corrects it by "relativistic or Einstenian effects" instead of starting with Einstein's gravitational theory right away.

\section{MAIN CHARACTERISTICS OF THE GPS SYSTEM}

Here we consider only the GPS system because the GLONASS is very similar. As it is well known, see ${ }^{1}, \underline{6}$, the GPS system is formed by three different parts or segments (see Fig. 1):

The nominal GPS operational constellation consists of 24 SVs that orbit the Earth in 12 
sidereal hours. There are often more than 24 operational satellites as new ones are launched to replace older satellites. Nowadays, for instance, the current GPS constellation consists of 29 satellites.

The orbit altitude is $20,183 \mathrm{~km}$, such that the satellites repeat the same track and configuration over any point (as the earth turns beneath them) approximately every 24 hours (4 minutes earlier each day). There are six orbital planes (with nominally four SVs in each), equally spaced (60 degrees apart), and inclined at about fifty-four degrees with respect to the equatorial plane.

The constellation provides the user or receiver with between five and eight SVs visible from any point on the Earth. Each satellite contains four atomic clocks, two of Cesium and two of Rubidium (precision of 4 nanoseconds per day), and they emit electromagnetic signals that include a code which indicates to the user its position with respect to the surface of the Earth in an ECEF system of reference and its GPS coordinate time which is advanced with respect to UTC (Universal Time Coordinated).

\section{2) Control Segment}

The Control Segment consists of four major components: 1.- The Master Control Station (MCS), located at Schriever Air Force Base (formerly Falcon AFB) in Colorado. 2.A Backup Master Control Station, located at Gaithersburg, Maryland. 3.- Four ground antennas that provide near real-time telemetry, tracking, and commanding interface among the GPS satellites and the MCS and 4.- Six Monitor quasi-equatorial stations around the world. These monitor stations measure signals from the SVs which are incorporated into orbital models for each satellite.

There are models that compute precise orbital data (ephemerides) and SV clock corrections for each satellite. The Master Control station uploads ephemerides and clock data to the SVs. The SVs then send subsets of the orbital ephemerides data to GPS receivers over radio signals.

\section{3) User Segment}

The GPS User Segment consists of the GPS receivers and the user community. GPS receivers convert SVs signals into position, velocity, and time estimates. Four satellites are required to compute the four coordinates of $\mathrm{X}, \mathrm{Y}, \mathrm{Z}$ (position) and $\mathrm{T}$ (time).

The position is computed with an error of 10 meters approximately (it depends on the positions of the SVs for a given location and time), although with the so-called differential 
techniques, the error can decrease to a few meters or even to a lower order. The idea behind differential positioning techniques (DGPS) is to correct bias errors at one location with measured bias errors at a known position. A reference receiver or base station computes corrections for each satellite signal. GPS receivers are used for navigation, positioning, time transfer, and other types of research.

\section{FOUR-DIMENSIONAL GEODESY}

The problem of positioning for a receiver on Earth (E) consists of calculating his spacetime position $\left(\mathbf{R}_{E}, T_{E}\right)$, from four spacetime events emitted by four satellites $\left(\mathbf{r}_{S_{j}}, t_{S j}\right), j=$ $1,2,3,4$.

The equations of navigation of the GPS are based on the application of the second principle of Special Relativity (constancy of the speed of light in vacuum), in usual Minkowskian coordinates, they are:

$$
\left|\mathbf{R}_{E}-\mathbf{r}_{S_{j}}\right|^{2}=c^{2}\left(T_{E}-t_{S_{j}}\right)^{2} ; j=1,2,3,4 .
$$

These are the equations of four light rays (electromagnetic signals) that travel on four light cones (3-dimensional hypersurfaces) from the satellites to the receiver in Minkowski's spacetime and are only valid in a local inertial reference frame in the presence of a gravity field. In the GPS, this reference frame is the Earth Centered local Inertial (ECI) reference system whose origin coincides with the Earth's center of mass. It is in free fall and does not turn with the Earth, i.e., it is non-rotating. This local inertial frame, ECI, is related by a coordinate transformation to the International Celestial Reference Frame (ICRF). The ICRF is a local inertial frame defined by the coordinates of 717 extragalactic radio sources and its origin is at the center of mass of the solar system.

From the point of view of the Newtonian reference systems, the first mission of the GPS is to determine the 3-dimensional position of the receiver on Earth (that is east-west, northsouth and vertical) in the ECEF reference system. In principle, the signals from three satellites provide this information. Each satellite sends a signal that codifies where the satellite is and the time of emission of the signal. The receiver clock times the reception of each signal, then subtracts the time of emission to determine the lapse of time travel and hence the distance that the signal has travelled from the SVs at the speed of light. 
This is the distance the satellite was from the receiver when it emitted the signal. Taking these three distances as radii, three spheres $S^{2}$ are constructed with center on each satellite. The receiver is located in one of the two intersection points of the three spheres $S^{2}$. This is, in short, the procedure of trilateration.

Nevertheless, there is still a problem: the receiver clock is usually made of quartz crystals oscillators and hence it is not as accurate as the atomic clocks in the SVs. For this reason, the signal of a fourth satellite is needed to obtain the error in the precision of the receiver clock and enables the user's clock to be used as an atomic clock.

We will see below that, from the point of view of the relativistic positioning systems, the receiver determines its four coordinates (in general since we will see, not necessarily three of coordinate space and one of coordinate time, but four proper times), identifying the points of intersection of the four light cones of the satellites that intersect the light cone of a receiver, see Fig. 2.

\section{RELATIVISTIC EFFECTS IN THE GPS}

In the GPS constellation, the satellite clocks keep time to an accuracy of about four nanoseconds per day ( $4 \mathrm{~ns} / \mathrm{d}=4 \times 10^{-9} \mathrm{~s} / \mathrm{d}$ ), which amounts to a fractional time stability of 5 parts in $10^{14}$. This means that once an atomic clock of in the SVs is set, then after one

day it should be correct to within about 4 nanoseconds. Therefore, the errors introduced by the relativistic effects at order $c^{-2}$ were crucial in its conception, because these effects are of the order of hundred or thousand nanoseconds. To ignore these effects would make the GPS useless for three main reasons ${ }^{7}$ :

\section{First. Einstein gravitational blue shift effect.}

The clocks run faster when they are far away from a center of gravitational attraction. This center is the center of the Earth in the case of GPS. Hence, there is a blue shift of the frequency for a signal sent downward from the satellites to the Earth, due to usual Newtonian gravitational effects depending on the distance to the center of the Earth. We stress that, in a time independent gravitational field (as the gravitational field of the Earth in first approximation) the coordinate frequency of the radio signal is conserved as it propagates, hence the blue shift of the photon at its reception on Earth is due to the fact that the time scale is slower for the Earth bound clock. 


\section{Second. Time dilation or special relativistic Doppler effect of second order.}

The motion of both the SVs and the Earth receivers must be taken into account. As the satellite clocks move faster than the clocks on the Earth's surface this gives rise, on the one hand to the classical Doppler effect (linear in speed), and to the other to the special relativistic Doppler effect of second order in the satellite speed $O\left(v_{S}^{2} / c^{2}\right)$ due to time dilation (clocks in motion run slow). Consequently, the latter gives place to a red shift of the frequency for a signal sent downward from the satellites to the Earth.

\section{Third. Sagnac effect and gravitomagnetic field of rotation.}

In principle, it is necessary to consider the Earth rotation for two reasons. Firstly, the kinematical Sagnac effect $\underline{\underline{8}-9}$, which is of order $c^{-2}$ and produces a maximum error of the order of $207.4 \mathrm{~ns} / \mathrm{d}$ in the GPS. Secondly, the effect of the gravitomagnetic field $(\mathrm{GM}) \underline{10}$ generated by the rotation of the Earth's mass. Even today the effects of this GM field have not been incorporated to GNSS because they are of order $O\left(c^{-3}\right)$, i.e. picoseconds, and hence negligible at the accuracy presently required.

The Schwarzschild metric appears to be sufficient to correct the relativistic effects at order $c^{-2}$, something which is necessary to describe the spacetime near the Earth. Nevertheless, strictly speaking, the static metric with spherical symmetry of Schwarzschild does not describe exactly the spacetime near the Earth for two reasons:

1) The Earth is not spherical.

2) The Earth's mass does not remain static but, when turning it generates a stationary field of rotational gravity (non-Newtonian) or gravitomagnetic field (GM). Thus, the external gravity field of the Earth can be described more adequately by a Kerr metric or its LenseThirring linear approximation.

However, the Earth rotates slowly with an angular velocity at the equator of $\omega_{E}=$ $7.2921151247 \times 10^{-5} \mathrm{rad} \mathrm{s}^{-1}$ or, equivalently, at a linear speed of $v_{E}=465 \mathrm{~m} \mathrm{~s}^{-1}$. Hence so far, with the required precision in the measurement of the time (ns), the Schwarzschild metric is a sufficient approximation for the objetives of the GNSS.

Let us remark nevertheless, that according to the IAU-2000 resolutions the gravitomagnetic effects, which have the order $O\left(c^{-3}\right)$, must be incorporated to define as the barycentric celestial reference system (BCRS) as well as the geocentric celestial reference system (GCRS). So, in Astrometry the gravitomagnetic effects must be considered because the order of accuracy is greater than in the current GNSS. 
In classical Droste-Hilbert coordinates the expression of the Schwarzschild metric for equatorial orbits, reads as:

$$
d s^{2}=c^{2} d \tau^{2}=\left(1-\frac{2 G M}{r c^{2}}\right) c^{2} d t^{2}-\left(1-\frac{2 G M}{r c^{2}}\right)^{-1} d r^{2}-r^{2} d \psi^{2}
$$

where $\tau$ is the local proper time of a comoving observer at rest, $t$ is far-away global time or coordinate time measured in an inertial frame placed at spatial infinity and $\psi$ is the azimuthal angle.

Let us consider a circular orbit (see note ${ }^{11}$ ), then the clock on the Earth and the satellite clock travel at constant distance around the Earth center, therefore $d r=0$ for each clock. For both clocks, one obtains:

$$
\frac{1}{c^{2}}\left(\frac{d s}{d t}\right)^{2}=\left(1+\frac{2 \phi}{c^{2}}\right)-\frac{v^{2}}{c^{2}}
$$

where $\phi$ is the Newtonian potential and $v=r \frac{d \psi}{d t}$ is the coordinate tangential speed along the circular equatorial orbit, measured using the coordinate far-away time $t$.

Now, let us apply Eq. (3) twice, first to the clock in a satellite (S), (using $r=r_{S}, v=v_{S}$ and proper time $d \tau_{S}=d s / c$ ) and secondly to a fixed clock in the Earth's equator and turning with it (E) (using, $r=r_{E}, v=v_{E}$ and proper time $d \tau_{E}=d s / c$ ), with the same elapsed coordinate time, $d t$, corresponding to an inertial observer at spatial infinity. Then, dividing both expressions one obtains:

$$
\left(\frac{d \tau_{E}}{d \tau_{S}}\right)^{2}=\frac{1-\frac{2 G M}{r_{E} c^{2}}-\frac{v_{E}^{2}}{c^{2}}}{1-\frac{2 G M}{r_{S} c^{2}}-\frac{v_{S}^{2}}{c^{2}}}
$$

The relativistic effects we are considering are only those of order $O\left(c^{-2}\right)$. This is the order of approximation used in the GPS. So, why is this order of approximation good enough? Because, as said above, the accuracy of the SVs atomic clocks is nanoseconds and only these three effects are of higher magnitude.

Are the relativistic effects considered small at this order? No, as we will show, they are not only important, but crucial.

On the other hand, it is important to point out that the necessity to consider other smaller relativistic effects at the order $O\left(c^{-3}\right)$ when laser cooled atomic clocks of last generation are used, with measurable errors of order of picoseconds, as in the ACES (Atomic Clock 
Ensemble in Space) mission of the ESA for the ISS13 , and those of the order $O\left(c^{-4}\right)^{14,15}$, when one works with intrinsic errors of femtoseconds in the near future.

\section{A. Einstein gravitational blue shift effect: 45,700 ns/d}

Now, let us suppose that we ignore the motions of the satellite clock and of the clock on the Earth's surface, i.e, let us start ignoring the Doppler effect of second order of Special Relativity, that is to say both are fixed in the ECI: $v_{E}=v_{S}=0$. Then, Eq. (4) reduces to

$$
\frac{d \tau_{E}}{d \tau_{S}}=\frac{\left(1-\frac{2 G M}{r_{E} c^{2}}\right)^{\frac{1}{2}}}{\left(1-\frac{2 G M}{r_{S} c^{2}}\right)^{\frac{1}{2}}} .
$$

The radius of a circular orbit of a SV with a period of 12 sidereal hours is approximately $r_{S}=26,561 \mathrm{~km}$. The linear approximation of (5) reads as:

$$
\frac{d \tau_{E}}{d \tau_{S}}=1-\frac{G M}{r_{E} c^{2}}+\frac{G M}{r_{S} c^{2}}=1-D .
$$

The corresponding numerical values for these terms are:

$$
\frac{G M}{r_{E} c^{2}}=6.95 \times 10^{-10}
$$

and

$$
\frac{G M}{r_{S} c^{2}}=1.67 \times 10^{-10}
$$

In Eq. (6) $D$ is a positive number and gives an estimate of the Einstein gravitational blue shift, between the stationary clocks in the position of satellite and on the Earth's surface. A displacement towards the blue is observed in the clocks on the Earth when a radio signal is sent downward from the satellites to Earth.

Is this difference negligible or is it crucial for the functioning of GPS?. Taking into account that in one nanosecond an electromagnetic signal travels about $30 \mathrm{~cm}$, we have that if a difference of hundreds of nanoseconds causes difficulties in the accuracy of the GPS, then a difference of thousands of nanoseconds like that which is obtained with the Einstein effect will cause even greater difficulties in the accuracy.

The clock in the satellite runs faster, i.e., it goes ahead about $45.7 \mu \mathrm{s} / \mathrm{d}$ compared with a clock on the Earth's surface, due to gravitational effects associated with position exclusively. General Relativity is clearly needed for the correct operation of the GPS. 


\section{B. Time dilation or special relativistic Doppler effect of second order: $7,100 \mathrm{~ns} / \mathrm{d}$}

Besides the gravitational effects of the position of the satellite and the observer on the Earth, we must also consider the effects of Special Relativity due to relative motion of the clocks in the satellite and of a clock fixed on the Earth's surface. The clock of the satellite moves at greater speed than a fixed clock on the Earth's surface. Their speeds are, $v_{S}=3.874 \mathrm{~km} / \mathrm{s}$, and $v_{E}=465 \mathrm{~m} / \mathrm{s}$, respectively. It is well known that Special Relativity predicts that "clocks in motion slow down" or, in other words, to an SV higher speed corresponds a red shift when the light is received on Earth.

Therefore, this kinematic Doppler effect of second order "works against" the Einstein gravitational blue shift effect. At the critical altitude of $H=3,167 \mathrm{~km}$ they compensate each other, but at the altitude of the satellites of the GPS, $H_{G P S}=20,183 \mathrm{~km}$, the Einstein gravitational blue shift effect is dominant.

The final formula that accounts for both effects is obtained from Eq. (4) which in the first order of approximation reads:

$$
\frac{d \tau_{E}}{d \tau_{S}}=1-\frac{G M}{r_{E} c^{2}}-\frac{v_{E}^{2}}{2 c^{2}}+\frac{G M}{r_{S} c^{2}}+\frac{v_{S}^{2}}{2 c^{2}} .
$$

An analogous formula (except for a change of sign) for circular equatorial orbits, was first given in ${ }^{16}$. Introducing numerical values in (9), the net result is of the order of 39,000 ns/d , which is equivalent to an error of $11,700 \mathrm{~m}$ after a day of operation.

\section{GPS coordinate time}

As we will see, the previous net result, in which only proper times appear, must be corrected using the so-called GPS coordinate time and a more realistic gravitational potential for the Earth. We restart with a more realistic metric for the weak external gravitational field of the Earth which is obtained from the linearized Schwarzschild metric added to the

gravitational potential of the mass quadrupole moment. The GPS uses this approximated metric to describe the spacetime near the Earth. This metric, when expressed in the ECI reads, to order $O\left(c^{-2}\right)$, as:

$$
d s^{2}=\left(1+\frac{2 V}{c^{2}}\right) c^{2} d t^{2}-\left(1-\frac{2 V}{c^{2}}\right) d r^{2}-r^{2} d \Omega^{2} .
$$


Here $V$ is the Newtonian potential $\phi$ plus the Newtonian quadrupole potential of the Earth; and where $d \Omega^{2}=d \theta^{2}+\sin ^{2} \theta d \psi^{2}$.

Nevertheless, the ephemerides of the satellites are calculated in the ECEF system. Thus, carrying out the transformation of coordinates from the ECI to the ECEF $\left(t=t^{\prime} ; r=\right.$ $\left.r^{\prime} ; \theta=\theta^{\prime} ; \psi=\psi^{\prime}+\omega_{E} t^{\prime}\right)$, the spacetime metric in the ECEF reference frame has the expression:

$$
\begin{aligned}
d s^{2}= & \left(1+\frac{2 \Phi_{\mathrm{eff}}}{c^{2}}\right) c^{2} d t^{2}-2 \omega_{E} r^{2} \sin ^{2} \theta d \psi^{\prime} d t \\
& -\left(1-\frac{2 V}{c^{2}}\right) d r^{2}-r^{2} d \Omega^{2},
\end{aligned}
$$

where the cross term in Eq. (11) gives rise to the kinematical Sagnac effect, $, \underline{9}, \frac{9}{}$, due to the rotation of the ECEF, and where $\Phi_{\text {eff }}$ is the effective potential in the ECEF rotating frame, which now contains also the centripetal potential, and reads:

$$
\Phi_{\text {eff }}=V-\frac{\omega_{E}^{2} r^{2} \sin ^{2} \theta}{2} .
$$

The equipotential condition $\Phi_{\text {eff }}=\Phi_{0}=$ const, defines the geoid model or reference ellipsoid of geodesists. The term $\Phi_{0} / c^{2}$, when it is evaluated at the equator $\left(r=r_{E}\right.$, $\theta=\pi / 2)$, has the form:

$$
\frac{\Phi_{0}}{c^{2}}=-\frac{G M}{r_{E} c^{2}}-\frac{J_{2} G M}{2 r_{E} c^{2}}-\frac{\omega_{E}^{2} r_{E}^{2}}{2 c^{2}}=-6.9693 \times 10^{-10},
$$

where the WGS-84 value of the quadrupole coefficient $J_{2}$ is $1.0826300 \times 10^{-3}$ and $\omega_{E}=$ $15 \operatorname{arcsec} / \mathrm{s}$.

Thus, the metric in the ECEF frame evaluated at the geoid has the expression:

$$
\begin{aligned}
d s^{2}= & \left(1+\frac{2 \Phi_{0}}{c^{2}}\right) c^{2} d t^{2}-2 \omega_{E} r^{2} \sin ^{2} \theta d \psi^{\prime} d t \\
& -\left(1-\frac{2 V}{c^{2}}\right) d r^{2}-r^{2} d \Omega^{2} .
\end{aligned}
$$

Now, notice that in order to define the speeds that appear in Eq. (3), the coordinate time $t$ of the Schwarzschild metric measured in an inertial reference frame at spatial infinity is used instead of the coordinate time $t_{\mathrm{GPS}}$ of clocks at rest in the geoid. The relationship between them, as it can be seen from Eq. (14), is:

$$
d t_{\mathrm{GPS}}=\left(1+2 \Phi_{0} / c^{2}\right)^{1 / 2} d t
$$


or to first order

$$
d t_{\mathrm{GPS}}=\left(1+\Phi_{0} / c^{2}\right) d t
$$

As it appears in Eq. (13),$\Phi_{0}$ is the effective potential of gravity on the geoid that includes the centripetal term and the quadrupole potential. Also, we have calculated in Eq. (13) the negative correction, $\Phi_{0} / c^{2}$, which is of the order of seven parts in $10^{-10}$. Thus, the GPS coordinate time $t_{\mathrm{GPS}}$, runs slow with respect to the coordinate time $t$ measured by an inertial observer at infinity.

This metric in the ECI local inertial reference frame, when the relation Eq. (15) is introduced in Eq. (10), reads as:

$$
d s^{2}=\left[1+\frac{2\left(V-\Phi_{0}\right)}{c^{2}}\right] c^{2} d t_{\mathrm{GPS}}^{2}-\left(1-\frac{2 V}{c^{2}}\right) d r^{2}-r^{2} d \Omega^{2} .
$$

As it can be seen from Eq. (14), the global GPS coordinate time $t_{\mathrm{GPS}}$ is, also, the proper time of the clocks at rest on the geoid where $\Phi_{\text {eff }}=\Phi_{0}$. It is a time referred to ECEF (advanced with respect to the UTC), but coincides with a fictitious time of a clock at rest in the ECI, see also $\frac{17}{}$. This one is a local inertial reference frame in which the navigation equations are applicable and the Poincaré-Einstein synchronization can be established globally within it $\frac{18}{}$.

The spacetime metric referred to the ECI, and given in (17) can be transformed into:

$$
d s^{2}=\left[1+\frac{2\left(V-\Phi_{0}\right)}{c^{2}}-\left(1-\frac{2 V}{c^{2}}\right) \frac{1}{c^{2}}\left(\frac{d r}{d t_{\mathrm{GPS}}}\right)^{2}-\frac{r^{2}}{c^{2}}\left(\frac{d \Omega}{d t_{\mathrm{GPS}}}\right)^{2}\right] c^{2} d t_{\mathrm{GPS}}^{2}
$$

The tangential speed of the satellite in the ECI (supposing circular orbits, $d r=0$ ) is:

$$
v_{S}=r \frac{d \Omega}{d t_{\mathrm{GPS}}}
$$

hence the proper time in the satellite, at order $O\left(c^{-2}\right)$, is

$$
d \tau_{S}=d s / c=\left[1+\frac{\left(V-\Phi_{0}\right)}{c^{2}}-\frac{v_{S}^{2}}{2 c^{2}}\right] d t_{\mathrm{GPS}}
$$

and, integrating along a trajectory $C$, the GPS coordinate time reads as:

$$
\int_{C} d t_{\mathrm{GPS}}=\int_{C} d \tau_{S}\left[1-\frac{\left(V-\Phi_{0}\right)}{c^{2}}+\frac{v_{S}^{2}}{2 c^{2}}\right]
$$

In this formula the five main sources of error appear: 
1) In $\Phi_{0}$. Three effects on the fixed clocks in the ECEF: monopole (the main term responsible of the global blue shift), terrestrial quadrupole potential and centripetal potential due to the Earth's rotation, $v_{E}^{2} / 2 c^{2}$, (Doppler of second order effect, blue shift).

2) In $V$. The Einstein gravitational effect due to the position of the satellite (redshift) and the terrestrial quadrupole potential.

3) In $v_{S}^{2} / 2 c^{2}$. The special relativistic effect (Doppler of second order) due to the orbital motion of the satellite (red shift).

Neglecting the Earth's quadrupole moment in $V$ (i.e. supposing Keplerian orbits, $V=\phi$ ) and assuming that the SVs orbits are circular $\left(v_{S}^{2}=G M / r\right)$, one obtains:

$$
\int_{C} d t_{\mathrm{GPS}}=\int_{C} d \tau_{S}\left(1+\frac{3 G M}{2 r c^{2}}+\frac{\Phi_{0}}{c^{2}}\right) .
$$

Numerically, the constant rate correction is:

$$
\frac{3 G M}{2 r c^{2}}+\frac{\Phi_{0}}{c^{2}}=-4.4647 \times 10^{-10}=-38.58 \mu \mathrm{s} / \mathrm{d} .
$$

The negative sign of the sum of the second and third terms in (22) means that a clock in orbit gains time relative to a clock on the Earth. Therefore, using the global GPS coordinate time $t_{\mathrm{GPS}}$, the clock in a satellite goes ahead with respect to an Earth fixed clock and the final net time gained is of the order of $38,580 \mathrm{~ns} / \mathrm{d}$, that is to say an error of $11,574 \mathrm{~m} / \mathrm{d}$. In fact, when the first GPS satellite equipped with a Cesium clock was launched in 1977, its relativistic correction program was not activated for 20 days, during that time its atomic clock provided a great test for relativity losing the figure that appears in (23).

So, for the satellite atomic clocks to appear to the observer in the ECEF at the operating frequency, $10.23 \mathrm{MHz}$, the atomic clocks of the satellites must be fitted before their launch, lowering their proper frequency to:

$$
\left[1-4.4647 \times 10^{-10}\right] \times 10.23 \mathrm{MHz}=10.22999999543 \mathrm{MHz}
$$

In this way, the orbiting clock becomes a global coordinate GPS clock and the local proper time of satellite clocks is not used.

In addition to the above mentioned effects, it is necessary to correct the path dependent kinematical Sagnac effect or lack of global synchronization (maximum error of $207.4 \mathrm{~ns}$ ) due to the rotation of the ECEF and the effect of eccentricity (because the orbits are not circumferences but ellipses). In the GPS (GLONASS), these two small effects of order $O\left(c^{-2}\right)$ 
are corrected by the receiver clock on the Earth (clock in the satellite). It is remarkable that one can use the GPS to measure the Sagnac effect as it was done in $\underline{19}$.

Other effects of order $c^{-2}$, as the secondary Sagnac effect due to the polar motion or the tidal effects of the Moon and Sun, are negligible in the GPS and GLONASS because their magnitude is in the order of picoseconds .

Finally it is necessary to indicate that, when transferring frequencies, the classical longitudinal Doppler effect (linear in speed), also appears which in principle must be accounted for by the receivers. Depending on the speed of the receiver, it is $10^{3}-10^{5}$ higher than the secular relativistic effects considered so far.

A more detailed study of relativistic effects of order $1 / c^{2}$ on the GPS can be found in $20,21,22,23$; the post-Newtonian effects of order $1 / c^{3}$, as for instance the Shapiro time delay, in $\frac{13}{}$, and those of order $1 / c^{4}$ in $\frac{14}{2}$. Finally, in $\frac{15}{}$, is considered the post-Minkowskian approximation to order $G^{2}$, which contains the post-Newtonian effect of order $1 / c^{5}$.

\section{THE SYPOR PROJECT FOR THE GALILEO}

\section{A. Basic idea}

The basic idea of this project which is expected to be accepted in a Framework Programme of the EU, as it is was reported in ${ }^{3}$ and ${ }^{4}$, is the following one: A constellation of SVs with clocks that interchange their proper time among themselves and with Earth receivers, is a fully relativistic system. In the SYPOR, the Segment of Control is in the constellation of satellites, see Fig. 3.

The function of this new Control Segment is not to determine the ephemerides of the satellites with respect to geocentric coordinates as in the Newtonian GNSS, but to determine the coordinates of the receivers with respect to the constellation of SVs. Therefore, the procedure used up to now in the Newtonian GNSS is inverted. The SYPOR would be composed of two sub-systems: An independent one, made up of the constellation of satellites (primary 4-dimensional positioning system) and another one, coupling the Earth to the first sub-system (terrestrial secondary 3-dimensional reference system, WGS84 or ITRF and a scale of time).

The main technical characteristics of the SYPOR are: 1) External control of the full 
system: it consists of a device in at least four of the SVs pointing to the ICRS (International Celestial Reference System). 2) Internal control of the parts of the system: a device in each satellite that exchanges its proper time with other SVs. 3) Control by the Segment of receivers: it consists of a device in each satellite that sends the proper times of its nearby satellites as well as its proper time to the Earth by means of electromagnetic signals.

The SYPOR is a long term project which is still in a state of theoretical construction. We

will now summarize its main conceptual basis. In Relativity, in the presence of gravity, the spacetime is modelled by a 4-dimensional Lorentzian manifold with a pseudo-Riemannian metric. So, for a domain of the manifold, there exists a large variety of mathematical coordinate systems but only a few number of them admit a physical interpretation and can be physically constructed by means of a protocol on a set of physical fields (point test particles, light rays, etc.).

\section{B. Reference systems and positioning systems}

Let us define location systems as the physical realizations of some coordinate systems. Location systems are of two different types: reference systems and positioning systems. The first ones are 4-dimensional reference systems which allow one observer, considered at the origin, to assign four coordinates to the events of his neighborhood by means of a transmission of information. Due to the finite speed of light this assignment is intrinsically retarded with a time delay. The second one are 4-dimensional positioning systems (as used in the SYPOR) which allow to every event of a given domain to know its proper coordinates without delay or in an immediate or instantaneous way.

In Relativity, a (retarded) reference system can be constructed starting from an (immediate) positioning system (it is sufficient that each event sends its coordinates to the observer at the origin) but not the other way around. In contrast, in Newtonian theory, 3-dimensional reference and positioning systems are interchangeable and as the velocity of transmission of information, the speed of light, is supposed to be infinite, the Newtonian reference systems are not retarded but immediate.

The reference and positioning systems defined here are 4-dimensional objects, including time location. This is not the common use, and so, the International Astronomical Union (IAU) still considers separately time scales and 3-dimensional reference systems such as the 
International Celestial Reference System (ICRS) or the International Terrestrial Reference System (ITRF) .

Following 24 , the best way to visualize and characterize a spacetime coordinate system is to start from four families of coordinate 3-surfaces, then, their mutual intersections give six families of coordinate 2-surfaces and four congruences of coordinate lines. Alternatively, one can use the related covectors or 1 -forms $\left\{\boldsymbol{\theta}^{i}\right\}, i=1,2,3,4$, instead of the 3 -surfaces, and the vectors of a coordinate tangent frame $\left\{\boldsymbol{e}_{i}\right\}, i=1,2,3,4$, instead of four congruences of coordinate lines which are their integral curves. In this way, for a specific domain of a Lorentzian spacetime, each coordinate system is fully characterized by its causal class, which is defined by a set of 14 characters:

$$
\left\{\mathrm{c}_{1} \mathrm{c}_{2} \mathrm{c}_{3} \mathrm{c}_{4}, \mathrm{C}_{12} \mathrm{C}_{13} \mathrm{C}_{14} \mathrm{C}_{23} \mathrm{C}_{24} \mathrm{C}_{34}, c_{1} c_{2} c_{3} c_{4}\right\} \text {, }
$$

being $c_{i}$ the Lorentzian causal character of the vector $\boldsymbol{e}_{i}$, i.e. if it is spacelike, timelike or lightlike; $\mathrm{C}_{\mathrm{ij}}$ the causal character of the adjoint 2-plane $\left\{\boldsymbol{e}_{i} \wedge \boldsymbol{e}_{j}\right\}$ and, finally, $c_{i}$ the causal character of the covectors $\boldsymbol{\theta}^{i}$ of the dual coframe, $\boldsymbol{\theta}^{i}\left(\boldsymbol{e}_{j}\right)=\delta_{j}^{i}$. The covector $\boldsymbol{\theta}^{i}$ is timelike (resp. spacelike) iff the 3 -plane generated by the three vectors $\left\{\boldsymbol{e}_{j}\right\}_{j \neq i}$ is spacelike (resp. timelike). This applies for both Newtonian and Lorentzian spacetimes. In addition, for the latter, the covector $\boldsymbol{\theta}^{i}$ is lightlike iff the 3 -plane generated by $\left\{\boldsymbol{e}_{j}\right\}_{j \neq i}$ is lightlike or null.

This new degree of freedom (lightlike) in the causal character, which is proper of Lorentzian relativistic spacetimes but which does not exist in Newtonian spacetimes, allows to obtain, after a deep algebraic study ${ }^{24}$, the following conclusion: In the 4-dimensional Newtonian spacetime there exist four, and only four, causal classes of frames, whereas in the relativistic 4-dimensional Lorentzian spacetime, due to the freedom commented above, there exists 199, and only 199, causal classes of frames.

As notation for the causal characters, we will use lower case roman types (s,t,l) to represent the causal character of vectors (resp. spacelike, timelike, lightlike), and capital types $(\mathrm{S}, \mathrm{T}, \mathrm{L})$ and lower case italic types $(s, t, l)$ to denote the causal character of 2-planes and covectors, respectively.

The ECI and ECEF Newtonian coordinate systems used in the GPS belong to two of the four causal classes of Newtonian frames, \{tsss,TTTSSS,tsss\} and $\{\mathrm{ttss}, \mathrm{TTTTTS}, s s s\}$, respectively. For instance, the ECI coordinate tangent frame is $\left\{\boldsymbol{e}_{1}=\partial / \partial t, \boldsymbol{e}_{2}=\partial / \partial r, \boldsymbol{e}_{3}=\partial / \partial \theta, \boldsymbol{e}_{4}=\partial / \partial \psi\right\}$ and the corresponding coframe is 
$\left\{\boldsymbol{\theta}^{1}=d t, \boldsymbol{\theta}^{2}=d r, \boldsymbol{\theta}^{3}=d \theta, \boldsymbol{\theta}^{4}=d \psi\right\}$.

In Relativity, a specific causal class, among the 199 ones, can be assigned to any of the different coordinate systems used in all the solutions of the Einstein equations. However, for the same coordinate system and the same solution, the causal class can change depending on the region of the spacetime considered.

A coordinate system can be constructed starting from a tangent frame or its coordinate lines. But in this way, in general, there are obstructions to obtain positioning systems that are generic, i.e., valid for many different spacetimes. On the other hand, generic positioning systems can be constructed from four one-parameter families of 3-surfaces or, equivalently, from a cotangent frame. One clock broadcasting its proper time is described in the spacetime by a timelike line in which each event is the vertex of a future light cone. The set of these light cones of a emitter constitutes a one-parameter (proper time) family of null hypersurfaces. So, four clocks broadcasting their proper times determine four one-parameter families of null or lightlike 3-surfaces.

In a relativistic spacetime, the wave fronts of those signals parameterized by the proper time of the clocks, define four families of light cones (3-dimensional null hypersurfaces) which contain all the lightlike geodesics of four emitters and making a contravariant null (or light) coordinate system $\stackrel{24}{ }$, see Fig. 2. Such a coordinate system does not exist in a Newtonian spacetime where the light travels at infinite speed.

\section{Coll positioning systems}

Among the 199 Lorentzian causal classes, only one is privileged to construct a generic (valid for a wide class of spacetimes), gravity free (the previous knowledge of the gravitational field is not necessary) and immediate positioning system. This is the causal class $\{\operatorname{siss}, \mathrm{SSSSSS}, l l l l\}$ of the Derrick-Coll-Morales coordinate system $\underline{25}, 26$. This class includes the emission coordinates of the Coll positioning systems $\underline{\underline{4}}, \underline{\underline{5}}$. A coordinate system of this class has associated four families of null 3-surfaces or a null coframe, whose mutual intersections give six families of spacelike 2-surfaces and four congruences of spacelike lines.

In this primary positioning system, an user at any event in a given spacetime region can know its proper coordinates. The four proper times of four satellites $\left(\left\{\tau^{A}\right\} ; A=1,2,3,4\right)$ read at an event by a receiver or user constitute the null (or light) proper emission coordinates 
or user positioning data of this event, with respect to four SVs, see Fig. 4. These four numbers can be understood as the "distances" between the event and the four satellites.

The 3-null surface $\tau^{A}=$ const, for each A, represents the set of events that read the proper time $\tau^{A}$ of an event of the satellite path. The coordinate lines, given by the intersection of three future light 3 -surfaces $\tau^{B}=$ const, $\forall B \neq A$, are the integral curves of the frame vectors $\partial / \partial \tau^{A}$. Every line in a light 3-surface is lightlike (along the generatrix) or spacelike. The conclusion is that $\partial / \partial \tau^{A}$ cannot be timelike. Moreover, a null 3-surface contains a unique null direction at each point. Hence, the intersection among three light 3-surfaces can include their null directions only if they coincide at the point, namely if the three light 3-surfaces are tangent to each other. But, in this case the Derrick-Coll-Morales coordinate system would be degenerated, therefore the coordinate lines are always spacelike.

In a certain domain $\Omega \subset \boldsymbol{R}^{4}$ of the grid of parameters $\left(\left\{\tau^{A}\right\} ; A=1,2,3,4\right)$, any user receiving continuously his emission coordinates may know his trajectory. If the receiver has his own clock, with proper time denoted by $\sigma$, then he can know his trajectory, $\tau^{A}=\tau^{A}(\sigma)$, and his four-velocity, $u^{A}(\sigma)=d \tau^{A} / d \sigma$.

It is worth remarking that a similar positioning system could be conceived for the whole Solar system by using four millisecond pulsars.

There is no spacetime asymmetry like in the usual ECI Newtonian coframe ( $t s s$ ) (one timelike " $t$ " and three spacelike " $s$ "). In emission coordinates obtained from a general real null coframe $(l l l l)=\left\{d \tau^{1}, d \tau^{2}, d \tau^{3}, d \tau^{4}\right\}$, which is neither orthogonal nor normalized, the contravariant spacetime metric is symmetric with null diagonal elements and it has the general expression ${ }^{27}$ :

$$
\left(g^{A B}\right)=\left(d \tau^{A} \cdot d \tau^{B}\right)=\left(\begin{array}{cccc}
0 & g^{12} & g^{13} & g^{14} \\
g^{12} & 0 & g^{23} & g^{24} \\
g^{13} & g^{23} & 0 & g^{34} \\
g^{14} & g^{24} & g^{34} & 0
\end{array}\right)
$$

where $g^{A B}>0$ for $A \neq B$. Four null covectors can be linearly dependent although none of them is proportional to another. To ensure that the four null covectors are linearly independent and span a 4-dimensional spacetime, it is sufficient that $\operatorname{det}\left(g^{A B}\right) \neq 0$. Finally, this metric has a Lorentzian signature $(+,-,-,-)$ iff $\operatorname{det}\left(g^{A B}\right)<0$. The expression (25) of the metric is observer independent and has six degrees of freedom. A splitting of this 
metric can be considered changing from the six independent components of $g^{A B}$ to a more convenient set, which neatly separates two parameters depending only on the direction of the covectors $d \tau^{A}$ from other four parameters depending on the length of the covectors. A special case of normalized covectors giving a regular tetrahedric configuration with one degree of freedom for the four SVs was also considered in 27,28 for a Minkowski spacetime.

The SYPOR is not only a positioning system, but it can also be made autonomous or autolocated. Let us define the meaning of autonomous or autolocated. A system is autonomous or autolocated if any receiver can determine its spacetime path as well as the trajectories of the four satellites, solely on the basis of the information received during a proper time interval.

Four satellites emitting, without the necessity of a synchronization convention, not only their proper times $\tau^{A}$, but also the proper times $\tau^{A B}$ of three close satellites received by the satellite $A$ in $\tau^{A}$ (in total $\left\{\tau^{A}, \tau^{A B}\right\} ; A \neq B ; A, B=1,2,3,4$ ), constitute an autonomous positioning system. This is because the three proper times, together with its proper time, that a satellite clock receives from other SVs constitute its proper emission coordinates. Note that, today, with the new generation of satellites in operation in the GPS, this procedure of proper time auto navigation with cross-links can be technically fulfilled.

The sixteen data $\left\{\tau^{A}, \tau^{A B}\right\}$ or emitter positioning data received by a user, which allows the user to know his trajectory and the trajectories of four SVs, constitute a local chart of the null contravariant coordinate system of the atlas obtained by means of the whole constellation of SVs. Recently in ${ }^{29}$ some explicit examples have been studied for the simpler 1+1-dimensional Minkowski case. If the constellation is located around the Earth, like in the Galileo, would be needed more than four satellites and the redundancies could be used to model the true gravitational field acting on the constellation.

\section{Positioning and gravimetry}

In General Relativity, the gravitational field is described by the spacetime metric. If this metric is exactly known a priori, the system just described will constitute an ideal positioning system (and the components of the metric could be expressed in Derrick-CollMorales coordinates). In practice, the true spacetime metric (i.e., the gravitational field) is

not exactly known (in the GPS it is supposed to be the Schwarzschild one) and the satellite 
system itself has to be used to infer it. This problem arises when a satellite system is used for both positioning and measuring the spacetime metric. This implies that the positioning problem and the gravimetry problem cannot be separated.

To solve this joint problem, the considered satellites should have more than one clock: they may carry an accelerometer providing more information on the spacetime metric (in fact on the spacetime connection). Of course, in first approximation the satellites are in freefall (i.e., follow a geodesic of the spacetime metric) and consequently have zero acceleration. However, we are considering here the realistic case where the acceleration is nonzero due, for instance, to a small drag in the high atmosphere and this is measured by the accelerometers. Also, the satellites may have a gradiometer, this would give additional information on the metric (in fact, on the Riemann tensor of the spacetime). With these informations (and perhaps some additional ones) an optimization procedure can be developed ( $\mathrm{sec}^{30,31}$ ) to obtain the "best observational metric field" and, thus, the best observational gravitational field acting on the constellation.

For the purposes of positioning alone, there is no need to use navigation equations as in the GNSS Newtonian systems because the emission coordinates are defined without any reference to an Earth based coordinate system and this allows to achieve maximum precision for the primary (Earth-surface independent) autolocated positioning system. The primary autolocated positioning system alone does not allow the users to situate themselves with respect to the Earth's surface but it nevertheless permits every user to situate himself with respect to the constellation of satellites or with respect to any more conventional reference system deduced from it and also allows two or more users to know their relative positions.

Of course, for applications on or near the Earth's surface, the primary emission coordinates should be attached to some terrestrial secondary 4-dimensional Newtonian coordinate system, like the 3-dimensional World Geodetic System reference system and the GPS coordinate time, as in the GPS, or for instance, to the 3-dimensional International Terrestrial Reference System (ITRF), which is also an ECEF, and to the International Atomic Time (TAI). The possibility appears for a space agency to concentrate its interest in the primary positioning system and delegate to Earth agencies the control of the terrestrial coordinates. The function of an Earth agency must be to provide the coordinate transformation between the primary (Earth-surface independent) 4-dimensional relativistic positioning system and the secondary (Earth-surface dependent) 4-dimensional Newtonian reference system. 
In summary, the purpose of the SYPOR project is to divide a GNSS in two hierarchical systems: first, a primary system (Earth-surface independent), made up of the constellation of satellites. Its physical realization implies an Inter Satellite Link between neighboring satellites, a device in each satellite to send to the Earth the links (physical local proper times) directly received by each satellite, and a device in some of the satellites of the system to connect the constellation of satellites to the 3-dimensional International Celestial Reference System (ICRS). Secondly, a secondary system (Earth-surface dependent), coupling an intrinsically retarded Earth 3-dimensional reference system (WGS 84 or ITRF) and a global conventional time, as the GPS one, to the 4-dimensional immediate primary main system.

\section{CONCLUSIONS}

The aim of this work was to offer a simple introduction to the relativistic effects on the current GNSS: GPS and GLONASS. These systems are based on a Newtonian model which is corrected numerically by important "relativistic effects". This model uses the ECI and ECEF "Newtonian" reference frames, respectively for time reference and navigation. The influence of Special and General Relativity on the GNSS is considered through the exposition of the main relativistic effects of time dilation and Einstein gravitational shift. It is shown that if these effects were not taken into account the GNSS would fail after only a tiny fraction of an hour. Expressed differently, the correct operation of the GNSS confirms Special Relativity and the weak field and slow motion limit of General Relativity.

On the other hand, a constellation of SVs with satellites that exchange their proper time among them and with Earth users, is a fully relativistic system. Hence, this allows to use a contravariant light coordinate system in a GNSS that does not exist in a Newtonian spacetime and so an autonomous positioning system can be conceived, as in the SYPOR project.

The application of the SYPOR project, would make of the Galileo project a fully relativistic autonomous positioning system. It would not be necessary to correct the errors that arise from the relativistic effects (one obtains the sum of the post-Newtonian series in $c^{-n}$ ) and it would have additional technological advantages. Finally, the satellite system could be used for both, positioning and measuring the gravitational field acting on the constellation. 


\section{Acknowledgments}

I am grateful to Bartolomé Coll by the ideas on the SYPOR project for the Galileo that he communicated to me. Also I thank A. San Miguel, M. del Mar San Miguel and F. Vicente for suggestions and a critical reading of this work. Finally, I am grateful to an anonymous referee for its comments. The author is currently partially supported by the grants of the Spanish Ministerio de Educación y Ciencia MEC ESP2006-01263, MCyT TIC2003-07020 and Junta de Castilla y León VA065A05.

* Electronic address: e-mail: jfpascua@maf.uva.es

1 http://tycho.usno.navy.mil/gps.html

2 http://www.glonass-ianc.rsa.ru/

3 B. Coll, Elements for a theory of relativistic coordinate systems. Formal and physical aspects. In: J.-F. Pascual-Sánchez, L. Floría, A. San Miguel, and F. Vicente, editors, Reference Frames and Gravitomagnetism, p. 53. World Scientific, Singapore, 2001.

4 B. Coll, Physical Relativistic Frames. In: N. Capitaine, editor, Journées 2001 Systèmes de Référence Spatio-Temporels. Observatoire Royal de Belgique, 2003.

5 B. Coll, A principal positioning system for the Earth. (2003) gr-qc/0306043.

6 P. Dana, http://www.colorado.edu/geography/gcraft/notes/gps/gps_f.html

7 J.-F. Pascual-Sánchez, Lecture given in Scientific Days 250 years of the Spanish Astronomy, held at Real Observatorio de la Armada, R.O.A., San Fernando, Cádiz (Spain), Sept. 22-26, 2003 gr-qc/0405100.

8 J.-F. Pascual-Sánchez, A. San Miguel, F. Vicente, Isotropy of the velocity of light and the Sagnac effect. In: G. Rizzi and M. L. Ruggiero, editors, Relativity in Rotating Frames, p. 167. Kluwer, Amsterdan, 2004, gr-qc/0305076.

9 N. Ashby, The Sagnac effect in the Global Positioning system. In: G. Rizzi and M. L. Ruggiero, editors, Relativity in Rotating Frames, p. 11. Kluwer, Amsterdan, 2004.

10 B. Mashhoon, Gravitoelectromagnetism. In: J.-F. Pascual-Sánchez, L. Floría, A. San Miguel, and F. Vicente, editors, Reference Frames and Gravitomagnetism, p. 133. World Scientific, Singapore, 2001 [gr-qc/0011014]. 
11 We remark that in the special case of circular orbits $d r=0$, the metric coefficient of spatial curvature $g_{r r}$, i.e., the prefactor of $d r^{2}$ in Eq. (2), has no influence on the result of Eq. (3). So, in this approximation we could start from a metric that comes from the Equivalence Principle (local equivalence of inertia and gravity for experiments not sensitive to tidal forces), that reads:

$$
d s^{2}=c^{2} d \tau^{2}=\left(1-\frac{2 G M}{r c^{2}}\right) c^{2} d t^{2}-d r^{2}-r^{2} d \psi^{2}
$$

and it is not necessary to begin from the full nonlinear Schwarzschild metric Eq. (2) or even from its linear approximation. To obtain the above metric only the part of the Einstein Equivalence Principle that relies on Local Position Invariance (LPI) is needed. LPI states (see $\underline{12}$ ) that the outcome of any local non-gravitational experiment is independent of where and when in the universe it is performed.

12 C. M. Will, Theory and Experiment in Gravitational Physics, Cambridge, C. U. P., 1993.

13 L. Blanchet, C. Salomon, P. Teyssandier, and P. Wolf, Relativistic theory for time and frequency transfer to order $c^{-3}$. Astron. Astrophys. 370, 320 (2001).

14 B. Linet, P. Teyssandier, Time transfer and frequency shift to the order $1 / c^{4}$ in the field of an axisymmetric rotating body. Phys. Rev. D 66, 024045 (2002).

15 Ch. Le Poncin-Lafitte, B. Linet, and P. Teyssandier, World function and time transfer: general post-Minkowskian expansions. Class. Quantum Grav. 21, 4463 (2004).

16 S. F. Singer, Application of an Artificial Satellite to the Measurement of the General Relativistic "Red Shift". Phys. Rev. 104, 11 (1956).

17 C. Lämmerzahl, Relativity and Technology. Ann. Phys. (Leipzig) 15, 5 (2006).

18 E. Minguzzi, A globally well-behaved simultaneity connection for stationary frames in the weak field limit. Class. Quantum Grav. 21, 4123 (2004).

19 D. Allan, N. Ashby, M. Weiss, Around-the-World Relativistic Sagnac Experiment. Science 228, 69 (1985).

20 N. Ashby, Relativity and the global positioning system. Physics Today 55, May 2002, p. 41.

21 N. Ashby, Relativity in the global positioning system. Living Rev. Relativ. 6, 1 (2003).

22 T. B. Bahder, Navigation in curved space-time. Am. J. Phys. 69, 315 (2001).

23 T. B. Bahder, Relativity of GPS Measurement. Phys. Rev. D 68, 063005 (2003).

24 B. Coll, J. A. Morales, 199 Causal Classes of Space-Time Frames. Intern. Jour. Theor. Phys. 31, 1045 (1992). 
25 G. H. Derrick, On a completely symmetric choice of space-time coordinates. J. Math. Phys. 22, $2896(1981)$.

26 B. Coll, J. A. Morales, Symmetric frames in Lorentzian spaces. J. Math. Phys. 32, 2450 (1991).

27 M. Blagojević, J. Garecki, F. W. Hehl, and Yu. N. Obukhov, Real null coframes in general relativity and GPS type coordinates. Phys. Rev. D 65, 044018 (2002).

28 C. Rovelli, GPS observables in general relativity. Phys. Rev. D 65, 044017 (2002).

29 B. Coll, J. J. Ferrando, and J. A. Morales, Two-dimensional approach to relativistic positioning systems. Phys. Rev. D 73, 084017 (2006).

30 A. Tarantola, L. Klimes, J. M. Pozo, and B. Coll, Gravimetry, Relativity and the Global Navigation Satellite Systems. Lecture given in International School on Relativistic Coordinates, Reference and Positioning Systems held at Salamanca (Spain), January 21-25, 2005. To be published in the Proceedings.

31 http://www3.usal.es/ ft/rc2005/index_english.html 